\title{
LOSS-INSENSITIVE VECTOR ENCODING WITH TWO-UNIFORM FRAMES
}

\author{
BERNHARD G. BODMANN AND VERN I. PAULSEN
}

\begin{abstract}
The central topic of this paper is the linear, redundant encoding of vectors using frames for the purpose of loss-insensitive data transmission. Our goal is to minimize the reconstruction error when frame coefficients are accidentally erased. Two-uniform frames are known to be optimal for handling up to two erasures, in the sense that they minimize the largest Euclidean error norm when up to two frame coefficients are set to zero. Here, we consider the case when an arbitrary number of the frame coefficients of a vector is lost. We derive general error bounds and apply these to concrete examples. We show that among the 227 known equivalence classes of two-uniform $(36,15)$-frames arising from Hadamard matrices, there are 5 that give smallest error bounds for up to 8 erasures.
\end{abstract}

\section{INTRODUCTION}

Today it is common practice with digital media that data is transmitted in packets of a fixed size, whereby the content of each packet is typically encoded according to some error correction protocol. In the situation studied in this paper, each packet contains $n$ coefficients that characterize a vector in a $k$-dimensional (real or complex) Hilbert space, with $k<n$ to ensure data recovery when some part of the coefficients are lost in the transmission (or impractically delayed). The coefficients are linear in the input, that is, the vector is encoded with a frame by taking its inner products with the frame vectors. While these $n$ frame coefficients are transmitted, partial data loss may occur resulting in one or more frame coefficients being set to zero. In this paper, we estimate the Euclidean reconstruction error due to a lossy transmission and evaluate the performance of various frames.

In practice, it is commonly assumed that losing one coefficient in the transmission process is rare, and that the occurence of two lost coefficients in one packet is much less likely. A similar hierarchy of probabilities usually holds for a higher number of lost coefficients. This

Key words and phrases. Frames, codes, erasures, graphs, two-graphs, Hadamard matrix, conference matrix, error bounds. 
motivates the design of frames for such transmission purposes following an inductive scheme: We require perfect reconstruction when no data is lost. Among the frames giving perfect reconstruction, we want to minimize the maximal error in the one-deletion case. Generally, we continue by choosing among the frames which are optimal for $m$-deletions those performing best for $m+1$-deletions.

Casazza and Kovacevic [4] proved that among all Parseval frames, the uniform ones minimize the worst-case Euclidean reconstruction error when one frame coefficient is set to zero. Holmes and Paulsen [12] showed that so-called two-uniform frames perform best in this worst case scenario with up to two erasures. Here, we investigate the behavior of two-uniform frames when more than two frame coefficients are erased.

\section{Terminology And Notation}

We fix our notation for frames and introduce a measure for the reconstruction error when $m$ frame coefficients are erased.

Definition 1. Let $\mathcal{H}$ be a real or complex Hilbert space of dimension $k$. We call a subset $F=\left\{f_{j}\right\}_{j=1}^{n} \subset \mathcal{H}$ of $n$ vectors a uniform $(\mathbf{n}, \mathbf{k})$ frame if the Parseval-type equality $\|x\|^{2}=\sum_{j=1}^{n}\left|\left\langle x, f_{j}\right\rangle\right|^{2}$ holds for every $x \in \mathcal{H}$ and if there is a constant $c>0$ with $\|f\|=c$ for all $f \in F$. If the vectors in $F$ have different norms, then the frame is no longer called uniform. The linear map $V$ defined by $(V x)_{j}=\left\langle x, f_{j}\right\rangle,\{j \in$ $1 \ldots n\}$ is called the analysis operator, and its Hermitian adjoint $V^{*}$ the synthesis operator.

Facts 2. According to some elementary matrix theory, $F$ is an $(n, k)$ frame if and only if the Grammian (or correlation) matrix $V V^{*}=$ $\left(\left\langle f_{j}, f_{i}\right\rangle\right)$ of the frame vectors is a self-adjoint $n \times n$ projection of rank $k$. Moreover, the rank of a projection is equal to its trace, so $\operatorname{tr}\left(V V^{*}\right)=k$. Thus, when $F$ is a uniform $(n, k)$-frame, each of the diagonal entries of $V V^{*}$ must be equal to $k / n$, and hence each frame vector $f_{j}$ must be of length $\left\|f_{j}\right\|=\sqrt{k / n}$.

Conversely, given an $n \times n$ self-adjoint projection $P$ of rank $k$, we can always factor it as $P=V V^{*}$ with an $n \times k$ matrix $V$, by choosing an orthonormal basis for the range of $P$ as the column vectors of $V$. It follows that $V^{*} V=I_{k}$ and hence $V$ is the matrix of an isometry and so corresponds to an $(n, k)$-frame. Moreover, if $P=W W^{*}$ is another factorization of $P$, then necessarily $W^{*} W=I_{k}$ and there exists a unitary $U$ such that $W^{*}=U V^{*}$ and hence the two corresponding frames differ by multiplication by this unitary. Thus, $P$ determines a 
unique unitary equivalence class of frames. A self-adjoint projection $P$ corresponds to a uniform $(n, k)$-frame if and only if all of its diagonal entries are $k / n$.

Suppose that in the process of transmission some number, say $m$, of the components of the vector $V x$ are lost, garbled or just delayed for such a long time that one chooses to reconstruct $x$ with what has been received. In this case we can represent the received vector as $E V x$, where $E$ is a diagonal matrix of $m 0$ 's and $n-m 1$ 's corresponding to the entries of $V x$ that are, respectively, lost and received. The 0 's in $E$ can be thought of as the coordinates of $V x$ that have been "erased" in the language of Ref. [9]. If we tried to recover the vector $x$ using the synthesis operator, then the reconstruction error would be $V^{*}\left(I_{n}-E\right) V x$. Therefore, we measure frame performance in terms of the error operators of the form $V^{*}\left(I_{n}-E\right) V$.

Definition 3. Let $\mathcal{D}_{m}$ denote the set of diagonal matrices with exactly $m$ diagonal entries equal to one and $n-m$ entries equal to zero.

Given an $(n, k)$-frame $F$, we set the maximal error $e_{m}^{\infty}(F)=$ $\max \left\{\left\|V^{*} D V\right\|: D \in \mathcal{D}_{m}\right\}$, where $V$ is the analysis operator of $F$, and by the norm of a matrix we always mean its operator norm.

Since the set $\mathcal{F}(n, k)$ of all $(n, k)$-frames is a compact set, the value

$$
e_{1}^{\infty}(n, k)=\inf \left\{e_{1}^{\infty}(F): F \in \mathcal{F}(n, k)\right\}
$$

is attained and we define the optimal 1-erasure frames to be the nonempty compact set $\mathcal{E}_{1}^{\infty}(n, k)$ of frames where this infimum is attained, i.e.,

$$
\mathcal{E}_{1}^{\infty}(n, k)=\left\{F \in \mathcal{F}(n, k): e_{1}^{\infty}(F)=e_{1}^{\infty}(n, k)\right\} .
$$

Proceeding inductively, we now set for $1 \leq m \leq n$,

$$
e_{m}^{\infty}(n, k)=\inf \left\{e_{m}^{\infty}(F): F \in \mathcal{E}_{m-1}^{\infty}(n, k)\right\}
$$

and define the optimal m-erasure frames to be the nonempty compact subset $\mathcal{E}_{m}^{\infty}(n, k)$ of $\mathcal{E}_{m-1}^{\infty}(n, k)$ where this infimum is attained.

For the purposes of distinguishing the encoding performance of a frame, the unitary equivalence relation of frames coming from factorizations of the projection $P=V V^{*}$ is too fine. Therefore, we define an equivalence relation with respect to the behavior under deletions.

Definition 4. We say that two frames $F$ and $G$ with analysis operators $V$ and $W$ are equivalent if $U V V^{*} U^{*}=W W^{*}$ for some $n \times n$ unitary $U$ that is the product of a permutation and a diagonal unitary (or diagonal orthogonal matrix, in the real case). 
Remark 5. If $V$ and $W$ belong to equivalent frames such that $U V V^{*} U^{*}=$ $W W^{*}$ for an appropriate unitary $U$, then for any $D \in \mathcal{D}_{m}$, we have $D=U^{*} D^{2} U$ and thus the norms $\left\|V^{*} D V\right\|=\left\|V^{*} U^{*} D^{2} U V\right\|=\left\|D U V V^{*} U^{*} D\right\|=$ $\left\|D W W^{*} D\right\|=\left\|W^{*} D W\right\|$. Consequently, the operator norm of $V^{*} D V$ is the same for all frames in one equivalence class.

Apart from simply ignoring the presence of deletions and always using the left inverse $V^{*}$ of $V$ for reconstruction, we could compute a left inverse for $E V$, supposing the deletions have been detected. The following proposition shows that this does not change which frames are considered optimal for encoding.

Proposition 6. Given an $(n, k)$-frame $F$ and a matrix $E$ with $m>0$ zeros and $n-m$ ones on the diagonal, such that a left inverse $L$ exists for $E V$, then the following three statements are equivalent: (1) The norm of the left inverse $L$ observes $\|L\| \leq\left\|L^{\prime}\right\|$, where $L^{\prime}$ is the leftinverse of any $E V^{\prime}$ belonging to another $(n, k)$-frame $F^{\prime}$. (2) The frame $F$ minimizes the norm of the operator $V^{*} E V$ over all $(n, k)$-frames. (3) The minimal eigenvalue of $T=|E V|$ is maximized among all $(n, k)$ frames.

Proof. If $E V$ has a left inverse, then the left inverse of minimum norm is given by $T^{-1} W^{*}$ where $E V=W T$ is the polar decomposition and $T=|E V|=\left(V^{*} E V\right)^{1 / 2}$. Thus, the minimum norm of a left inverse is given by $t_{\text {min }}^{-1}$ where $t_{\text {min }}$ denotes the least eigenvalue of $T$.

In the second alternative, the error in reconstructing $x$ is given by

$$
x-V^{*} E V x=V^{*}(I-E) V x=\left(I-T^{2}\right) x=V^{*} D V x
$$

where $D$ is a diagonal matrix of $m 1$ 's and $n-m 0$ 's. Thus, the norm of the error operator $V^{*} D V$ is $1-t_{m i n}^{2}$.

Hence we see that, when a left inverse exists, the problems of minimizing the norm of a left inverse over all frames and of minimizing the norm of the error operator over all frames are really equivalent and are both achieved by maximizing the minimal eigenvalue of $T$.

We note that a left inverse will exist if and only if the norm of the error operator $V^{*} D V$ is strictly less than one. In this paper, we study the norms of error operators rather than those of the left inverses, since this seems to lead to cleaner formulas. Our goal is to describe the frames for which the norms of these error operators are in some sense minimized, independent of which erasures occur. 


\section{Frames And GraphS}

We begin by recalling a result concerning the optimality of frames analogous to Casazza, Kovacevic, Holmes and Paulsen.

Definition 7. We call $F$ a 2-uniform $(\mathbf{n}, \mathbf{k})$-frame provided that $F$ is a uniform $(n, k)$-frame and in addition, the operator norm $\left\|V^{*} D V\right\|$ is constant for all $\mathrm{D}$ in $\mathcal{D}_{2}$.

Theorem 8. [12] If a 2-uniform $(n, k)$-frame $F$ exists among the uniform frames, then for $m \geq 2$, every frame in $\mathcal{E}_{m}^{\infty}(n, k)$ is 2-uniform and $e_{2}^{\infty}(n, k)=k / n+c_{n, k}$. If there does not exist a 2-uniform $(n, k)$-frame, then $e_{2}^{\infty}(n, k)>k / n+c_{n, k}$.

In the remainder of this section we study the existence and construction of 2-uniform frames. For many possible values of $(n, k)$, there do not exist any 2-uniform frames. Moreover, when there do exist 2-uniform frames, then there are at most finitely many such frame equivalence classes and hence the problem of determining optimal $\mathrm{m}$ erasure frames is reduced to the problem of finding representatives for each equivalence class and determining which one of these finitely many equivalence classes is optimal. We exploit a connection between graph and frame theory to construct error bounds for 2-uniform frames when $m \geq 2$ erasures occur.

Holmes and Paulsen derived that $F$ is a 2-uniform $(n, k)$-frame, if and only if $V V^{*}$ is a self-adjoint rank $k$ projection that can be written in the form $V V^{*}=a I+c_{n, k} Q$ where $a=k / n, c_{n, k}=\sqrt{\frac{k(n-k)}{n^{2}(n-1)}}$, and the so-called signature matrix $Q=\left(q_{i, j}\right)$ is a self-adjoint $n \times n$ matrix satisfying $q_{i, i}=0$ for all $i$ and for $i \neq j,\left|q_{i, j}\right|=1$.

Definition 9. Given a graph $G$ on $n$ vertices, the Seidel adjacency matrix of $G$ is defined to be the $n \times n$ matrix $A=\left(a_{i, j}\right)$ where $a_{i, j}$ is -1 when $i$ and $j$ are adjacent, it is +1 when $i$ and $j$ are not adjacent, and 0 when $i=j$.

The following result, summarized from the results in Ref. [12], explains the relationship between signature and adjacency matrices.

Theorem 10. [12] An $n \times n$ matrix $Q$ is the signature matrix of a real 2-uniform $(n, k)$-frame if and only if it is the Seidel adjacency matrix of a graph with 2 eigenvalues and in this case, $k$ is the multiplicity of the largest eigenvalue. Moreover, if $\left\{F_{i}\right\}, i \in\{1,2\}$, is a set of real 2-uniform frames, with associated signature matrices $\left\{Q_{i}\right\}$ and the corresponding graphs $\left\{G_{i}\right\}$, then $F_{1}$ and $F_{2}$ are frame equivalent if and only if $G_{1}$ and $G_{2}$ are switching equivalent graphs. 
Example 11. The dimension and codimension 1 case.

Let $J_{n}$ denote the $n \times n$ matrix all of whose entries are 1 . Then $Q=$ $J_{n}-I_{n}$ satisfies $Q^{2}=J_{n}^{2}-2 J_{n}+I_{n}=(n-2) J_{n}+I_{n}=(n-1) I_{n}+(n-2) Q$ and the degeneracy of the largest eigenvalue is $k=1$, yielding the rather uninteresting 2-uniform frame for the one dimensional Hilbert space.

However, $Q=I_{n}-J_{n}$ is also a signature matrix with $k=n-1$, which shows that for each $k$ there exists a 2-uniform $(k+1, k)$-frame.

This frame is described in detail in ref. [4] and is in fact the only real uniform $(k+1, k)$-frame, up to some natural equivalence. We shall refer to these examples, which exist for every $n$ as the trivial 2-uniform frames.

Example 12. Conference Matrices.

The idea of using conference matrices to construct frames of this type originates in Ref. [18].

$A$ real $n \times n$ matrix $C$ with $c_{i, i}=0$ and $c_{i, j}= \pm 1$ for $i \neq j$ is called a conference matrix [6] provided $C^{2}=(n-1) I$.

Thus, every symmetric conference matrix is a signature matrix with $k=n / 2$. So, in particular such matrices must be of even size and they yield real 2-uniform $(2 k, k)$-frames, for certain values of $k$.

Conference matrices are known to exist for many values of n. Paley[15] constructs symmetric conference matrices, for every $n=p^{j}+1 \equiv 2(\bmod$ 4) with p prime. For further examples, see Ref. [7].

If $C=-C^{t}$ is a skew-symmetric conference matrix, then setting $Q=i C$ yields a complex 2-uniform $(2 k, k)$-frame. Similarly, examples of skew-symmetric conference matrices can be found in many places in the literature. See, for example Refs. [19] and [7]. Note that conference matrices yield 2-uniform frames with redundancy 2. Conversely, it is not hard to see that the signature matrix of any real 2-uniform frame of redundancy 2 is a conference matrix.

Example 13. Hadamard Matrices.

Using Hadamard matrices to construct 2-uniform frames has been discussed in [12]. A real $n \times n$ matrix $H$ is called a Hadamard matrix [6] provided that $h_{i, j}= \pm 1$ and $H^{*} H=n I$. If $H=H^{*}$ is a symmetric Hadamard matrix and in addition, $h_{i, i}=1$ for all $i$, then $H$ is called a graph Hadamard. In this case $Q=H-I$ is a signature matrix for a real 2-uniform frame with $k=\frac{n+\sqrt{n}}{2}$.

Similarly, $Q=I-H$ is a signature matrix for a real 2-uniform frame with the degeneracy of the largest eigenvalue being $k=\frac{n-\sqrt{n}}{2}$.

The fact that in the real case, $Q$ must be a matrix of 0 's, 1 's and -1 's shows that for fixed $(n, k)$ there are only finitely many possibilities 
for the Grammian matrix of a 2-uniform $(n, k)$-frame. Consequently, up to equivalence, there can be only finitely many 2 -uniform $(n, k)$ frames for each pair $(n, k)$. Thanks to the discovery by [18] of the connection between equiangular frames and the earlier work of Seidel and his collaborators in graph theory, much of the work of on existence, construction and determining frame equivalence classes for these frames is already known and exists in the literature.

By referring to this literature, we can give a complete list of all integers $n \leq 50$ for which such graphs (and hence 2-uniform frames) are known to exist, together with information about how many frame equivalence classes there are in each case.

This information is gathered together in Table 1. When an integer $j$ appears in the column labeled, "frame equivalence classes", it indicates that exactly $j$ inequivalent real 2 -uniform $(n, k)$-frames exist. When the symbol $j+$ appears, it indicates that at least $j$ inequivalent real 2 -uniform $(n, k)$-frames are known to exist, but it is not known yet if this is exhausts all equivalence classes. The letters $C, H$ and $G$ in the column labeled "type" indicate that the corresponding frames are all constructed using conference matrices, graph Hadamards, or only arise from certain graphs, respectively.

So for example, using Table 1 , and looking at $n=36$, we see that there exist at least 227 switching inequivalent graph Hadamard matrices and these can be used to construct at least 227 frame inequivalent 2-uniform $(36,15)$-frames and at least 227 frame inequivalent 2-uniform $(36,21)$-frames. For $n=276$, there exists a graph whose Seidel adjacency matrix has exactly 2 eigenvalues, but it is neither a conference matrix nor graph Hadamard matrix, and this matrix can be used to construct a 2 -uniform $(276,23)$-frame that up to frame equivalence is the unique such frame.

Given $n$, Seidel [16] exhibits a one-to-one correspondence between the two-graphs on the set of $n$ elements and the switching equivalence classes of graphs on $n$ elements and gives a concrete means, given the two-graph, to construct a graph from the corresponding switching class. This opens the possibility of finding two-uniform frames by numerical enumeration of two-graphs and subsequent construction of adjacency matrices belonging to the corresponding switching classes.

\section{Graphs And ERror Bounds}

In this section we derive estimates and formulas for $e_{m}^{\infty}(F)$ when $F$ is a real 2-uniform frame, using connectivity properties of the graph associated to the signature matrix $Q$ of $F$. 
TABLE 1. Real 2-Uniform Frames

\begin{tabular}{|c|c|c|c|}
\hline No. of vectors $n$ & Dimension $k$ & No. of Equiv. Classes & Type \\
\hline 6 & 3 & 1 & $\mathrm{C}$ \\
10 & 5 & 1 & $\mathrm{C}$ \\
14 & 7 & 1 & $\mathrm{C}$ \\
16 & $6 ; 10$ & 1 & $\mathrm{H}$ \\
18 & 9 & 1 & $\mathrm{C}$ \\
26 & 13 & 4 & $\mathrm{C}$ \\
28 & $7 ; 21$ & 1 & $\mathrm{G}$ \\
30 & 15 & $6+$ & $\mathrm{C}$ \\
36 & $15 ; 21$ & $227+$ & $\mathrm{H}$ \\
38 & 19 & $11+$ & $\mathrm{C}$ \\
42 & 21 & $18+$ & $\mathrm{C}$ \\
46 & 23 & $80+$ & $\mathrm{C}$ \\
50 & 25 & $18+$ & $\mathrm{C}$ \\
176 & $22 ; 154$ & 1 & $\mathrm{G}$ \\
276 & $23 ; 253$ & 1 & $\mathrm{G}$ \\
\hline
\end{tabular}

Recall that if $F$ is a real 2 -uniform $(n, k)$-frame, then the Grammian $P$ of $F$ is an $n \times n$ matrix that is a projection of rank $k$ and has the form $P=k / n I+c_{n, k} Q$, where $c_{n, k}=\sqrt{\frac{k(n-k)}{n^{2}(n-1)}}$ and $Q$ is the Seidel adjacency matrix of a certain graph that we shall denote $G_{F}$.

We also need to recall a few concepts from graph theory.

Definition 14. A graph on $m$ vertices is called complete bipartite provided that the vertex set can be divided into two disjoint subsets, $V_{1}$ and $V_{2}$, of sizes, say $m_{1}$ and $m_{2}$ with $m_{1}+m_{2}=m$, such that no pair of vertices in $V_{1}$ or in $V_{2}$ are neighbors, but every vertex in $V_{1}$ is adjacent to every vertex in $V_{2}$. We shall denote this graph by $B\left(m_{1}, m_{2}\right)$. In our definition of complete bipartite graph, we allow the possibility that one of the sets is empty, so that the complete bipartite graph, $B(0, m)$, is the graph on $m$ vertices with no edges. If $G$ is a graph with vertex set $V$ and $W \subset V$ then by the induced subgraph on $W$, we mean the graph with vertex set $W$ and two vertices in $W$ are adjacent if and only if they were adjacent in $G$.

Note that if $F$ is a real 2-uniform $(n, k)$-frame with signature matrix $Q$ and graph $G_{F}$, then the Seidel adjacency matrix of an induced subgraph on $m$ vertices is just the $m \times m$ matrix obtained by compressing $Q$ to the corresponding entries.

We are grateful to Ryan Pepper for the following observation, which can also be found in the work of Seidel. 
Lemma 15. A graph on $m$ vertices is switching equivalent to the graph with no edges if and only if it is complete bipartite.

Proof. Given any complete bipartite graph corresponding to a preselected partition of vertices into two sets, we show that it can be obtained by switching the empty graph on $m$ vertices. Without loss of generality we may order the vertices $\left\{v_{1}, v_{2}, \ldots v_{m}\right\}$ such that the partition is given by $\left\{v_{j}\right\}_{j \leq r}$ and $\left\{v_{j}\right\}_{j>r}$, with $0 \leq r \leq m$. Let us choose the switching matrix

$$
S=\left(\begin{array}{cc}
+I_{r} & 0 \\
0 & -I_{m-r}
\end{array}\right)
$$

The empty graph on $m$ vertices has the Seidel adjacency matrix $J_{m}-I_{m}$, so that of the switched graph is then

$$
S(J-I) S=\left(\begin{array}{cc}
J_{r}-I_{r} & -J_{r, m-r} \\
-J_{m-r, r} & J_{m-r}-I_{m-r}
\end{array}\right)
$$

which by inspection belongs to the preselected complete bipartite graph. Moreover, switching the empty graph always leads to complete bipartite graphs. Again, the empty (edgless) graph is in our sense bipartite, corresponding to a partition $\emptyset$ and $\left\{v_{1}, v_{2}, \ldots v_{m}\right\}$.

Theorem 16. Let $F$ be a real 2-uniform $(n, k)$-frame. Then $e_{m}^{\infty}(F) \leq$ $k / n+(m-1) c_{n, k}$ with equality if and only if $G_{F}$ contains an induced subgraph on $m$ vertices that is complete bipartite.

Proof. The proof has three parts. First we show that the operator norm $\left\|D V V^{*} D\right\|$ is equal to the largest eigenvalue of the compression of $V V^{*}$ to the rows and columns where $D$ has 1's. Then we bound the largest possible eigenvalue. Finally we characterize the case when this bound is saturated.

To begin with, we note that $D V V^{*} D$ is a positive operator, and so is its compression $\left(V V^{*}\right)_{m}$ to the rows and columns where $D$ has 1 's. Consequently, the operator norm of $D V V^{*} D$ is equal to the largest eigenvalue of $\left(V V^{*}\right)_{m}$. This eigenvalue, in turn, follows from the largest eigenvalue of the compression of $Q$, because $\left(V V^{*}\right)_{m}=\frac{k}{n} I_{m}+c_{n, k} Q_{m}$. So we can reduce the calculation to that of the largest eigenvalue of $Q_{m}$. In fact, to simplify the argument, we will look for the largest possible eigenvalue of $Q_{m}+I_{m}$ and later adjust for the added constant.

We now claim that the largest eigenvalue of $Q_{m}+I_{m}$ occurs when $Q_{m}+I_{m}=J_{m}$, that is, the matrix of all 1's. This follows from considering that for any given $x \in \mathbb{R}^{m}$ and $Q_{m}$, changing signs to make all their entries positive increases $\left\langle x,\left(Q_{m}+I_{m}\right) x\right\rangle /\|x\|^{2}$. 
By inspection, the largest eigenvalue of $J_{m}$ is $m$, so that of $J_{m}-I_{m}$ is $m-1$, and the claimed error bound follows:

$$
\left\|D V V^{*} D\right\| \leq \frac{k}{n}+(m-1) c_{n, k}
$$

To characterize cases of equality, suppose $G$ contains an induced subgraph that is switching equivalent to the graph with no edges. If we choose $D$ to have 1 's in the places on the diagonal corresponding to the vertices of this subgraph and 0's everywhere else, then $D(I+Q) D$ is switching equivalent to $D J_{n} D$ and so the error is $e_{m}^{\infty}(F)=k / n+(m-$ 1) $c_{n, k}$. Conversely, assume that equality holds in the error estimate. Then, $\|D(I+Q) D\|=m$. Given an eigenvector $x$ corresponding to eigenvalue $\pm m$, we may choose a switching matrix $S$ such that all of the entries of $S x$ are positive. Similarly to the above reasoning, all the entries in $S(I+Q) S$ must be 1's in the rows and columns where $D$ has 1's on the diagonal, otherwise it would be possible to increase the largest eigenvalue of $S D(I+Q) D S$ by flipping signs in $Q$, contradicting that the inequality is saturated. Hence, the induced subgraph on these vertices is switching equivalent to the edgeless graph.

Corollary 17. For a given $m$, a real 2-uniform frame $F$ maximizes the error $e_{m}^{\infty}(F)$ iff $G_{F}$ has an induced subgraph on $m$ vertices that is compelete bipartite.

Corollary 18. Let $F$ be a real 2-uniform $(n, k)$-frame. Then either $G_{F}$ contains an induced complete bipartite graph on 3 vertices or it is switching equivalent to the complete graph on $n$ vertices. Consequently, if $k<n-1$ we have $e_{3}^{\infty}(F)=k / n+2 c_{n, k}$.

Proof. Let us assume that $G_{F}$ has no induced complete bipartite subgraph on 3 vertices. We may choose one vertex and switch the others if necessary in order to have edges between this one and all others. Then any two vertices must be adjacent, otherwise there would be an induced complete bipartite subgraph on 3 vertices. Thus, the resulting graph is a switched version of $G_{F}$ that is complete. This corresponds to $F$ being equivalent to the uniform $(n, n-1)$-frame.

Corollary 19. If $F_{1}$ and $F_{2}$ are real 2-uniform $(n, k)$-graphs, then $e_{3}^{\infty}\left(F_{1}\right)=e_{3}^{\infty}\left(F_{2}\right)$.

By analogy with our earlier definitions, we call a Parseval frame $F$ 3-uniform if it is 2-uniform and if the error $\left\|D V V^{*} D\right\|$ associated with a deletion is constant for every $D \in \mathcal{D}_{3}$.

Corollary 20. The trivial 2-uniform $(n, k)$-frames, corresponding to $k=1$ or $k=n-1$, are 3-uniform. Conversely, if $F$ is a real 3-uniform 
$(n, k)$-frame, then either $k=1$ or $k=n-1$ and it is equivalent to the corresponding trivial frame.

Proof. It is clear from their definition that the trivial frames are 3uniform. What we need to show is that if $F$ is 3 -uniform then $G_{F}$ is either switching equivalent to the complete or to the edgeless graph.

To begin with, we pick a vertex and switch the others if necessary in order to isolate it. Any two additional vertices are either adjacent or not, and computing the norm of $D V V^{*} D$, where $D$ is associated with these 3 vertices, distinguishes these two cases. However, 3-uniformity then implies that every additional pair of vertices must behave the same way. Thus, if there is one edgeless induced subgraph on 3 vertices, then all of $G_{F}$ is edgeless. On the other hand, if there is one neighboring pair, then all pairs of vertices except those including the isolated one are neighbors. Switching this one vertex then yields the complete graph.

We now discuss how non-existence of complete bipartites gives rise to refined error bounds.

Definition 21. Let $\mathcal{G}_{m}^{(s)}$ denote the set of graphs on $m$ vertices such that $s$ is the minimum number of edges occuring among graphs in the equivalence class associated with each $G \in \mathcal{G}_{m}^{(s)}$.

We note that $\mathcal{G}_{m}^{(0)}$ are the complete bipartites on $m$ vertices, and $\mathcal{G}_{m}^{(1)}$ is the equivalence class of graphs that may be reduced to one edge by switching. However, for larger values of $s, \mathcal{G}_{m}^{(s)}$ may contain more than one equivalence class.

Lemma 22. Let, $Q^{(0)}, Q^{(1)}$ and $Q^{(s)}, s \geq 1$, be Seidel adjacency matrices belonging to graphs $G^{(0)} \in \mathcal{G}_{m}^{(0)}, G^{(1)} \in \mathcal{G}_{m}^{(1)}$, and $G^{(s)} \in \mathcal{G}_{m}^{(s)}$, respectively, for some common number of vertices $m \geq 3$. Denote by $\lambda^{(0)}, \lambda^{(1)}$ and $\lambda^{(s)}$ the largest eigenvalue of $Q^{(0)}, Q^{(1)}$ and $Q^{(s)}$. Then $\lambda^{(0)} \geq \lambda^{(1)} \geq \lambda^{(s)}$.

Proof. By appropriate switching, we can make $G^{(0)}, G^{(1)}$ and $G^{(s)}$ have a minimal number of edges in their respective equivalence class. In particular, then $G^{(0)}$ is the edgeless graph. Permuting the vertices if necessary, we have $G^{(0)} \subset G^{(1)} \subset G^{(s)}$. To simplify notation, we can choose this permutation in such a way that there is an edge between the $m$-th and $m-1$-th vertex in $G^{(1)}$ and in $G^{(s)}$. Since switching corresponds to a change of basis in $\mathbb{R}^{n}$, the maximal eigenvalues of the Seidel adjacency matrices $Q^{(0)}, Q^{(1)}$ and $Q^{(s)}$ are unchanged. After switching, the components of $Q^{(1)}$ and $Q^{(s)}$ observe $q_{m, m-1}^{(1)}=q_{m-1, m}^{(1)}=$ 
$q_{m, m-1}^{(s)}=q_{m-1, m}^{(s)}=-1$. The inequality between the largest eigenvalues of $Q^{(0)}$ and $Q^{(1)}$ follows by explicit computation, $\lambda^{(0)}=m-1 \geq \lambda^{(1)}=$ $\frac{m}{2}-2+\sqrt{\frac{m^{2}}{4}+m-3}$ for $m \geq 3$.

To establish the inequality $\lambda^{(1)} \geq \lambda^{(s)}$, we use a variational argument similar to that in the proof of Theorem 16. We consider a normalized eigenvector $x$ belonging to the largest eigenvalue of $Q^{(s)}$. We show there is a normalized vector $p$ such that $\lambda^{(1)} \geq\left\langle p, Q^{(1)} p\right\rangle \geq\left\langle x, Q^{(s)} x\right\rangle=\lambda^{(s)}$. The construction of $p$ proceeds in several parts:

Part 1. First let us assume that not all of $\left\{x_{i}\right\}_{i=1}^{m-2}$ are negative, otherwise we multiply $x$ by -1 . Now we set $x_{i}^{\prime}=\left|x_{i}\right|$ for $i \leq m-2$ and replace $q_{i, j}$ by $q_{i, j}^{\prime}:=\left|q_{i, j}\right|$ for the block given by $i, j \leq m-2$. At the same time, we modify the last two rows and columns of $Q^{(s)}$ as follows. If $x_{m-1}$ and $x_{m}$ are both positive or both negative, we set $p_{i}=\left|x_{i}\right|$ for all $i$, let $q_{m-1, j}^{\prime}=q_{j, m-1}^{\prime}=1$ and $q_{m, j}^{\prime}=q_{j, m}^{\prime}=1$ for $j \leq m-2$, and thus obtain $Q^{(1)}=Q^{\prime}$ from $Q^{(s)}$ while only increasing $\left\langle x, Q^{(s)} x\right\rangle$ to $\left\langle p, Q^{\prime} p\right\rangle$, which finishes the proof for this case.

Part 2. If one component of $x$, say $x_{m}$, is negative, we set $x_{i}^{\prime}=\left|x_{i}\right|$ only for $i \leq m-1$ and retain $x_{m}^{\prime}=x_{m}$, while letting $q_{i, m}^{\prime}=q_{m, i}^{\prime}=-1$ if $x_{i}<0$ and $q_{i, m}^{\prime}=q_{m, i}^{\prime}=1$ if $x_{i}>0$, for $i \leq m-2$. This ensures that $\left\langle x^{\prime}, Q^{\prime} x^{\prime}\right\rangle \geq\left\langle x, Q^{(s)} x\right\rangle$, while we have reduced the occurence of -1 's in $Q^{\prime}$ to the last row and column. Note that by our assumption that not all $\left\{x_{i}\right\}_{i=1}^{m-2}$ are negative, according to this procedure, there remains at least one entry $q_{i, m}^{\prime}=q_{m, i}^{\prime}=1$ in the last row and column of $Q^{\prime}$.

Part 3. Now define $x^{\prime \prime}$ by $x_{i}^{\prime \prime}=\left|x_{i}\right|$ and let $Q^{\prime \prime}$ be obtained from switching signs in the last row and column of $Q^{\prime}$. Then $\left\langle x^{\prime}, Q^{\prime} x^{\prime}\right\rangle=\left\langle x^{\prime \prime}, Q^{\prime \prime} x^{\prime \prime}\right\rangle$. Since $x^{\prime \prime}$ has all positive entries and $Q^{\prime \prime}$ contains at least one pair of -1 's in the last row and column, setting all entries to 1 but this one pair $q_{i, m}^{\prime \prime}=q_{m, i}^{\prime \prime}=-1$ only increases $\left\langle x^{\prime \prime}, Q^{\prime \prime} x^{\prime \prime}\right\rangle$ and transforms $Q^{\prime \prime}$, together with a permutation of indices, to $Q^{(1)}$. Applying the same permutation to the entries of $x^{\prime \prime}$ yields $p$ satisfying $\left\langle p, Q^{(1)} p\right\rangle \geq\left\langle x, Q^{(s)} x\right\rangle$.

Theorem 23. Given a real 2-uniform $(n, k)$-frame $F$ such that for some $m \geq 3$, the associated graph $G_{F}$ does not have an induced complete bipartite subgraph on $m$ vertices. Then we have the improved error bound

$$
e_{m}^{\infty}(F) \leq \frac{k}{n}+c_{n, k}\left(\frac{m}{2}-2+\sqrt{\frac{m^{2}}{4}+m-3}\right) .
$$

If $G_{F}$ contains an induced subgraph on $m$ vertices that differs from a complete bipartite by one edge, then equality holds. 
Proof. The improved error bound results from the fact that by the preceding lemma, in the absence of complete bipartites on $m$ vertices, the graphs in $\mathcal{G}_{m}^{(1)}$ maximize the error.

To prepare the argument, we recall that $V V^{*}=\frac{k}{n} I_{n}+c_{n, k} Q$ is a projection, so the compression of $D V V^{*} D$ to the rows and columns where $D$ has 1's, henceforth denoted as $\frac{k}{n} I_{m}+c_{n, k} Q_{m}$, is a non-negative operator. Consequently, the norm of $D V V^{*} D$ equals that of $\frac{k}{n} I_{m}+$ $c_{n, k} Q_{m}$ and is given by its largest eigenvalue. To obtain this eigenvalue, it is enough to consider $Q_{m}$.

In the absence of complete bipartites, we know that any matrix $Q_{m}$, obtained from the compression of $Q$ to $m$ rows and columns, corresponds to a graph $G \in \mathcal{G}_{m}^{(s)}$ with $s \geq 1$. By the inequality in the preceding lemma, to bound the largest possible eigenvalue we only need to consider $s=1$. Since we may switch and permute $G$ without loss of generality, we again choose $Q_{m}$ to be the $m \times m$ matrix of all 1's, except the 0 's on the diagonal and the two elements $q_{m-1, m}=q_{m, m-1}=-1$. By inspection, $Q_{m}$ has an eigenvector $(0,0, \ldots, 1,-1)$ with eigenvalue 1 , a set of $m-3$ linearly independent eigenvectors of eigenvalue -1 given by $(1,-1,0, \ldots, 0)$ and those obtained when exchanging its second entry with all others except the first one and the last two entries. The larger of the remaining two eigenvalues is $\lambda=\frac{m}{2}-2+\sqrt{\left(\frac{m}{2}-1\right)^{2}+2 m-4}$ which is seen to be greater than or equal to 1 for $m \geq 3$. The claimed error bound follows.

As a consequence of such bounds, the performance of a frame can be evaluated easily by numerical enumerative techniques in graph theory. Now we apply these error bounds to concrete examples. We compute $e_{m}^{\infty}$ for the real 2-uniform $(26,13)$-frame and show that it cannot handle 7 or more erasures, demonstrating a tradeoff in optimal performance between few and many erasures. As final example, we show that among the 227 known equivalence classes of two-uniform $(36,15)$ frames arising from Hadamard matrices, there are 5 that give smallest error bounds for up to 8 erasures.

Example 24. The 2-uniform frame of highest redundancy.

Among the known graphs giving rise to 2-uniform frames, the frame with highest redundancy is the 2-uniform $(276,23)$-frame that arises from the unique regular two-graph on 276 vertices [8]. This frame has redundancy 12. Applying the inequalities of the previous section we see that $e_{m}^{\infty}(F) \leq \frac{m+4}{60}$ for all $m$ and since the graph cannot contain any induced complete bipartite subgraphs on 23 or more vertices, this inequality must be strict for $m>23$. From this formula it follows that 
if any set of 55 or fewer erasures occurs, then a left inverse for $E V$, $L$, can be constructed with $\|L\| \leq \sqrt{60}$.

From these inequalitites it follows that given any subset $E$ of $F$ containing at most 56 frame vectors, the set $F \backslash E$ will still span $\mathbb{R}^{23}$. Since we do not precisely know the value of $e_{m}^{\infty}(F)$ it is possible that this frame can handle much larger sets of erasures. By comparison, if we had produced a frame by simply repeating an orthonormal basis 12 times, then that frame would be able to handle at most subsets of 11 erasures. On the other hand, by the argument given in the above remark, a generic uniform $(276,23)$-frame should be able to handle sets of up to $253 \mathrm{era}$ sures, but at the expense of having a larger value for $e_{2}^{\infty}(F)$.

We now turn our attention to the special case of graph Hadamards. Suppose $H$ is a graph Hadamard, which means $H=H^{*}, H^{2}=n I, \mathrm{H}$ contains elements $h_{i j}= \pm 1$ only, and the diagonal is fixed by $h_{j j}=$ 1. The following two results about error bounds of frames related to Hadamard matrices are based on an argument of Penny Haxell.

Proposition 25. Any real 2-uniform $(n, k)$-frame $F$ belonging to a signature matrix $Q=H-I$ with a graph Hadamard $H$ satisfies $e_{m}^{\infty}(F)=$ $\frac{k}{n}+(m-1) c_{n, k}$ if $n \geq 48$ and $m \leq 5$.

Proof. By conjugating $H$ by a diagonal matrix of \pm 1 's, we may always assume that the first row and column of $H$ consist entirely of +1 's. Then $H^{2}=n I$ implies that the column vectors of $H$ are orthogonal and thus every additional row and column has to have an equal number of +1 's and -1 's. Moreover, for any two columns other than the first, say $i$ and $j$, their orthogonality forces them to have $n / 4$ entries in common where both are +1 's, $n / 4$ entries in common where both columns are -1 's, $n / 4$ entries in common where column $i$ is +1 's and column $j$ is -1 's and $n / 4$ entries in common where column $i$ is -1 's and column $j$ is +1 's.

The claimed values for the error now follows from showing the existence of induced complete bipartite subgraphs on $m$ vertices in the graph $G$ associated with the signature matrix $Q=H-I$. After switching as described above, we see that $G$ contains one isolated vertex and that all other vertices have $n / 2$ neighbors. So let us pick as $v_{1}$ the isolated vertex and as $v_{2}$ any other, and as $v_{3}$ one vertex from those $\frac{n}{2}-2$ that are neither adjacent with $v_{1}$ nor $v_{2}$. Then by the orthogonality argument, $v_{3}$ has $n / 4$ neighbors that are adjacent with $v_{2}$ and a set of $n / 4$ neighbors in the set of $\frac{n}{2}-2$ that are not adjacent with $v_{1}$ or $v_{2}$. Thus, there remains a set $A$ of $\frac{n}{4}-3$ vertices that are not adjacent with $v_{1}, v_{2}$, or $v_{3}$. If in this set there is a pair of vertices that 
are not adjacent then we have found an induced edgeless subgraph on 5 vertices, the one consisting of $v_{1}, v_{2}, v_{3}$ and the additional two nonadjacent vertices in $A$. Thus, before switching, the subgraph induced by these vertices was complete bipartite.

If there is no non-adjacent pair in $A$, then the induced subgraph on the vertices in $A$ is a complete graph. We want to argue that this is impossible for $n$ sufficiently large. Note that $H$ has eigenvalues $\pm \sqrt{n}$ because $H^{2}=n I$. If $Q$ contains an induced complete subgraph of $s=\frac{n}{4}-3$ vertices, then the associated signature matrix $Q_{s}=I_{s}-J_{s}$ has eigenvalues 1 and $1-s$ and these must lie between those of $Q$, $-\sqrt{n}-1 \leq 1-s \leq 1 \leq \sqrt{n}-1$. Thus, it is impossible that $A$ induces a complete subgraph in $G$ when $n>28+8 \sqrt{6}>47.5$.

Proposition 26. Any real 2-uniform $(n, k)$-frame $F$ belonging to a signature matrix $Q=I-H$ with a graph Hadamard $H$ satisfies $e_{m}^{\infty}(F)=$ $\frac{k}{n}+(m-1) c_{n, k}$ if $n \geq 30$ and $m \leq 5$.

Proof. The first steps of the proof parallel the one for the preceding proposition, the only difference being that after switching to obtain the isolated vertex $v_{1}$, the valency of the other vertices is $\frac{n}{2}-2$. Having chosen a vertex $v_{2}$ and a vertex $v_{3}$ that is not adjacent with $v_{1}$ or $v_{2}$, we observe that there remains a set $A$ of $s=\frac{n}{4}-2$ vertices that are not adjacent with any of $v_{1}, v_{2}$, and $v_{3}$. As before, we obtain that $Q_{s}=I_{s}-J_{s}$ has eigenvalues 1 and $1-s$ if $A$ induces a complete subgraph of $s$ vertices in $G$, and then necessarily $1-\sqrt{n} \leq 1-s$, thus there cannot be such an induced complete subgraph if $n>16+8 \sqrt{3}>29.8$.

Remark 27. The smallest possible values of $n$ for graph Hadamards are $n \in\{4,16,36,64\}$. The preceding results imply that the graphs related to 2-uniform $(n, k)$-frames of Hadamard type are guaranteed to contain induced complete bipartites on 5 vertices for $n=36, k=15$ and any $n \geq 64$.

Example 28. The 227 known switching equivalence classes of graph Hadamards with $n=36$.

For $n=36, k=21$, the argument in Proposition 25 does not guarantee the existence of induced complete bipartite subgraphs on 5 vertices. However, by having a computer search all 227 known equivalence classes [17], one finds that all members have at least one induced complete bipartite subgraph on 6 vertices. Thus, the $m$-deletion error is the same for all 2-uniform $(36,21)$-frames,

$$
e_{m}^{\infty}(F)= \begin{cases}\frac{7+(m-1)}{12} & \text { if } m \leq 6 \\ 1 & \text { if } m>6\end{cases}
$$


Thus, if five or fewer erasures occur, then there exists a left inverse of norm at most $\sqrt{12}$.

If $n=36, k=15$, then Proposition 26 shows that each graph $G_{F}$ contains an induced complete bipartite on 5 vertices. Moreover, an explicit search finds that the maximal number of vertices that induce a complete bipartite subgraph varies from 6 to 8 among the 227 switchingequivalent classes: There are 217 switching-equivalent classes that have an induced complete bipartite subgraph on 8 vertices, 5 classes that have one on 7 vertices but not on 8 , and 5 classes that have one on 6 but not on more than 6 vertices. Thus, for the group of 217, we have that $e_{m}^{\infty}(F)=\frac{5+(m-1)}{12}$ for $m \leq 8$ and $e_{m}^{\infty}(F)=1$ for $m \geq 8$. For the next group of 5 equivalence classes that have an induced 7 -vertex complete bipartite subgraph, we have $e_{m}^{\infty}(F)=\frac{5+(m-1)}{12}$ if $m \leq 7$, while $e_{8}^{\infty}(F) \leq \frac{7}{12}+\frac{1}{12} \sqrt{21} \approx 0.965$. The last bound follows from Theorem 23 . Finally, for those having a maximal number of 6 vertices that induce a complete bipartite subgraph, we have $e_{m}^{\infty}(F)=\frac{5+(m-1)}{12}$ for $m \leq 6$, $e_{7}^{\infty}(F)=\frac{13}{24}+\frac{1}{24} \sqrt{65} \approx 0.878$ and $e_{8}^{\infty}(F) \leq \frac{7}{12}+\frac{1}{12} \sqrt{21} \approx 0.965$. Again, the results for the cases $m=7$ and $m=8$ follow from Theorem 23, because for each member of the group, one finds induced subgraphs on 7 vertices that differ from complete bipartites by only one edge, and we know that there are no induced complete bipartites on 8 vertices. The induced subgraphs giving the largest 8-deletion error are all found to be switching equivalent and related to complete bipartites by flipping two edges. Accordingly, the numerical value for the error $e_{8}^{\infty}(F) \approx 0.927$ for the members of this group is below the error bound derived from the absence of complete bipartites.

Thus, if 7 or fewer erasures occur, we know that a left inverse of $E V$ with norm at most $2 \sqrt{6} / \sqrt{11-\sqrt{65}} \approx 2.86$ exists, compared to $\sqrt{12} \approx$ 3.46 for the other 222 switching-equivalent classes. If 8 erasures occur, we know a left inverse exists of norm at most $\sqrt{12} / \sqrt{5-\sqrt{21}} \approx 5.36$.

To summarize, any 2-uniform $(36,15)$-frame belonging to the last group of 5 equivalence classes is better than the other 222 , because it will have smaller error bounds, but we cannot guarantee that it can handle any more than 8 erasures.

We list a representative of the signature matrices belonging to each of these optimal 5 equivalence classes in Table 2 .

Acknowledgments. This research was partially supported by the following grants: NSF grants DMS-0300128 and DMS-0406748 and University of Houston TLCC funds. 
TABLE 2. Signature matrices for optimal 2-uniform $(36,15)$-frames.
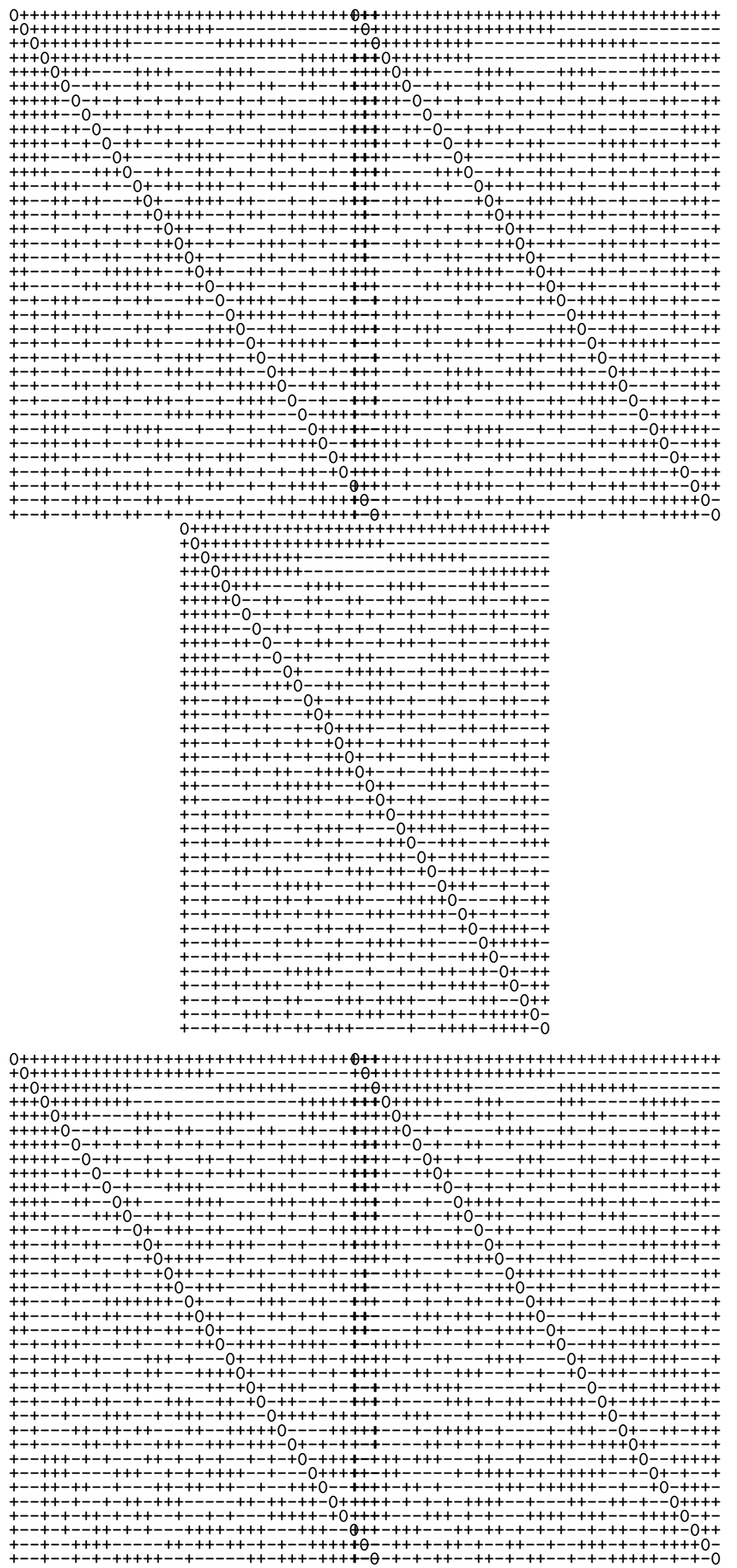


\section{REFERENCES}

[1] J.J. Benedetto and M. Fickus, "Finite normalized tight frames," Advances in Computational Mathematics 18, pp. 357-385, 2003.

[2] F.C. Bussemaker, R.A. Mathon and J.J. Seidel, "Tables of Two-Graphs," Combinatorics and Graph Theory, Lecture Notes in Mathematics 885 (S.B. Rao, ed.), pp. 70-112, 1981.

[3] F.C. Bussemaker and J.J. Seidel, "Symmetric Hadamard matrices of order 36," Ann. New York Acad. Sci. 175, pp. 66-79, 1970.

[4] P.G. Casazza, J. Kovacevic, "Equal-norm tight frames with erasures," Advances in Computational Mathematics 18, pp. 387-430, 2003.

[5] J.H. Conway, R.H.Hardin and N.J.A. Sloane, "Packing Lines, Planes, etc.: Packings in Grassmannian Spaces," Journal of Experimental Mathematics 5, pp. 139-159, 1996.

[6] J.H. Conway and N.J.A. Sloane, Sphere Packings, Lattices and Groups, 3rd Edition, Grundlehren der mathematischen Wissenschaften, no. 290, SpringerVerlag, 1999.

[7] J.M. Goethals and J.J. Seidel, "Orthogonal matrices with zero diagonal," Canad. J. Math. 19, pp. 1001-1010, 1967.

[8] J.M. Goethals and J.J. Seidel, "The regular two-graph on 276 vertices," Discrete Mathematics 12, pp. 143-158, 1975.

[9] V.K. Goyal, J. Kovacević, and J.A. Kelner, "Quantized frame expansions with erasures," Appl. Comput. Harmon. Anal. 10, no.3, pp. 203-233, 2001.

[10] V.K. Goyal, M. Vetterli, and N.T. Thao, "Quantized overcomplete expansions in $R^{n}$ : analysis, synthesis, and algorithms," IEEE Trans. Inform. Theory $\mathbf{4 4}$, no. 1 , pp. 16-31, 1998.

[11] R.B. Holmes, Optimal Frames, PhD Thesis, University of Houston, 2003.

[12] R.B. Holmes and V.I. Paulsen, "Optimal frames for erasures," Linear Algebra and its Applications 377, pp. 31-51, 2004.

[13] J.R. Holub, "Pre-frame operators, Besselian frames, and near-Riesz bases in Hilbert spaces," Proc. Amer. Math. Soc. 122, pp. 779-785, 1994.

[14] J.R. Holub, "The equivalence of frames," Bull. Polish Acad. Sci., Math. 45, pp. 73-76, 1997.

[15] R.E.A.C. Paley, "On orthogonal matrices," J. Math. Phys., 12, pp. 311-320, 1933.

[16] J.J. Seidel, "A survey of two-graphs," Proc. Intern. Coll. Teorie Combinatorie, (Roma 1973), Accad. Naz. Lincei, Roma, pp. 481-511, 1976.

[17] E. Spence, "Strongly regular graphs on at most 64 vertices," June 2003, available at www.maths.gla.ac.uk/ es/srgraphs.html .

[18] T. Strohmer and R. Heath, "Grassmannian frames with applications to coding and communication," Appl. Comp. Harm. Anal., 14, pp. 257-275, 2003.

[19] J. Williamson, "Hadamard's determinant theorem and the sum of four squares," Duke Math. J. 11, pp. 65-81, 1944.

[20] G. Zimmermann, "Normalized tight frames in finite dimensions," Recent Progress in Multivariate Approximation (K. Jetter, W. Haufmann and M. Reimer, eds.), Birkhäuser, pp. 249-252, 2001. 
LOSS-INSENSITIVE VECTOR ENCODING WITH TWO-UNIFORM FRAMES19

Department of Applied Mathematics, University of Waterloo, Waterloo, Ontario N2L 3G1, Canada

E-mail address: bgb@math.uwaterloo.ca

Department of Mathematics, University of Houston, 651 Phillip G. Hoffman Hall, Houston, TX 77204-3008, USA

E-mail address: vern@math.uh.edu 